Methods A rapid syphilis test (RST) was introduced into STD services among patients attending STD clinics and existing outreach services to female sex workers (FSW) in Liuzhou and Jiangmen, China to test the feasibility of same-day test and treatment (STAT) and same-day test and referral (STAR) strategies. The STAT and STAR rates were evaluated regarding the different STD providers and outreach teams, respectively.

Results Of 7283 patients from 3 STD clinics in Liuzhou-one public health clinic, one private reproductive health clinic and one general hospital clinic who were screened with a non-treponemal test, TRUST, and RST for syphilis, 291 (4.0\%) had active syphilis, but only $69.8 \%$ of infected cases accepted the STAT. The acceptance rate was different between clinics, with $54.2 \%$ in public health clinic, $71.8 \%$ in reproductive health clinic and $100.0 \%$ in general hospital clinic. During the outreach services provided by the CDC intervention team (CIT), STD clinic team (SCT) and reproductive health clinic team (RCT), 75\% of FSWs accepted to be tested with RST in sex work venues. Of 2780 FSWs screened with RST, 199 (7.2\%) were positive and were referred to designated clinics for further testing and treatment. However, success in referring RST positive FSWs to designated clinics were significantly different between outreach teams. RCT had the highest referral rate (85.7\%) followed by SCT (70.6\%) and CIT $(26.7 \%)$. Focus groups discussions among providers and highrisk populations regarding the introduction of RST indicated that social and cultural contexts were related to the successful introduction of RST among these populations. Trust of the target population in the clinics and outreach team was related to the acceptability of STAT and STAR. Belief in the results of a free RST test, confidentiality of syphilis status, social stigma, medical cost at the designated clinics for further test and treatment, and time spent for clinic visit were the main concerns of FSWs, especially in the referral advice.

Conclusions Feasibility in introduction of STAT and STAR among high-risk groups is encouraging but the barriers still exist. Social and cultural contexts are important and should be considered when RST is introduced as one of strategies for prevention and control of syphilis in China.

\section{S2.4 NEW TECHNOLOGIES TO SUPPORT STI SCREENING AND SYNDROMIC MANAGEMENT IN RESOURCE-LIMITED SETTINGS}

doi:10.1136/sextrans-2011-050102.8

R Peeling. London School of Health and Tropical Medicine, London, UK

Background High quality tests for detection of STIs are available but they are neither affordable nor available to patients in resourcelimited settings. For patients presenting with STI symptoms, WHO recommends the use of syndromic management in settings where laboratory services are not available. As most STI patients tend to have few or no symptoms, tests to screen for STIs is important for guiding treatment and prevention of onward transmission. However, in resource-limited settings, patients often have to travel long distances to reach a clinic with STI services, and many fail to return for test results and treatment after the initial visit.

Methods In recent years, increased investment in the development of novel diagnostic technologies has resulted in advances that have made it possible to provide STI-testing at all healthcare settings. The development of multiplex tests to support screening and STI syndromic management in resource-limited settings is an urgent priority.

Results Exciting new technologies may soon transform how STIs can be diagnosed in resource-poor settings in the next few years. Among them are duplex antigen/antibody tests that can be used for STI syndromes and rapid lateral flow tests that can be used with oral fluid instead of blood for syphilis and HIV. Nucleic acid amplified tests that are designed as integrated platforms providing sample- in- answer-out convenience and random access will soon be available for the detection of genital chlamydial and gonococcal infections. Simple isothermal nucleic acid amplification technologies are available for detection of bacterial and viral STIs in 15-60 min, using simple equipment that can be run on solar energy or batteries. A mathematical model developed by Aledort et al (2006) estimated that a test for syphilis that requires no laboratory infrastructure could save more than 201000 adjusted lives and avert 215000 stillbirths. A similar test could save approximately 4 million DALYs, avert more than 16.5 million incident gonorrhoea and chlamydial infections and prevent more than 212000 HIV infections.

Conclusions Recent investments in the development of point-of-care diagnostics for infectious diseases have produced novel technologies that can support STI screening and syndromic management in resource-limited settings. Quality assurance and implementation research are needed to ensure optimal uptake and full impact of these novel technologies in resource-limited settings.

\section{Symposium 3: Evaluation of large-scale and complex public health interventions S3.1 CHALLENGES AND KEY ISSUES IN EVALUATION OF PUBLIC} doi:10.1136/sextrans-2011-050102.9

L Dandona. Public Health Foundation of India, India

Interest in evaluating the impact of large-scale public health interventions has been increasing globally over the past few years to inform effective use of public resources. Consequently some highprofile evaluations of complex public health interventions to improve population health in low- and middle-income countries have been reported recently. This increased, and desirable, focus on evaluations has helped bring attention to some major challenges that are encountered when attempting to assess the impact of largescale public health interventions. These include the process of planning or commissioning of the evaluation, the design and analytical approaches that are both robust and feasible, and the interpretation and dissemination of findings. Examples will be used to illustrate these challenges. The key issues that need to be dealt with for improving evaluations of large-scale and complex public health interventions in low- and middle-income countries and better utilisation of the findings will be discussed.

\section{S3.2 THE ROLE OF COMMUNITY BASED RANDOMISED TRIALS IN THE EVALUATION OF PUBLIC HEALTH INTERVENTIONS}

doi:10.1136/sextrans-2011-050102.10

A Donner. University of Western Ontario, Ontario, Canada

In this talk I discuss methodological issues that arise in trials in which intact communities are allocated to each of several intervention groups. The issues discussed include the choice between a randomised and non-randomised design, the need to avoid selection bias when community members are sub-sampled, and the challenges involved in maintaining study power. Ethical issues unique to community intervention trials are also discussed. 